\title{
Aerodynamic Design on China New High-Speed Trains
}

\author{
Guowei Yang \\ Key Laboratory of High-Temperature Gas Dynamics, \\ Institute of Mechanics of CAS, Beijing 100190, China \\ gwyang@imech.ac.cn
}

\begin{abstract}
The high-speed trains have very complex running environments, which contain single-train running in open air, two-trains passing by in open air, singletrain running in tunnel and two-trains passing by in tunnel. As the environment wind appears, cross-wind effect must be considered. Aerodynamic design of highspeed trains mainly aims at the drag, lift, moment, impulse pressure waves, aerodynamic noise etc at typical running conditions. In the paper, the aerodynamic design processes of $\mathrm{CRH} 380 \mathrm{~A}$ and $380 \mathrm{~B}$ are introduced and the aerodynamic performances of different designs are emphatically analyzed and compared. Wind tunnel experiments and running tests indicate that the new generation of highspeed trains holds excellent aerodynamic performances.
\end{abstract}

\section{Introduction}

Since 2004, the manufacturing technologies and product lines of high-speed trains have successively introduced into China from the different high-speed railway developed countries, which contain CRH1, CRH2, CRH3 and CRH5 four highspeed trains. In order to satisfy the requirements of China high-speed railway network, the new generation of high-speed trains of CRH380A and CRH380B, based on the Japan and Germany technologies of CRH2 and CRH3, was decided to be studied and produced, whose maximum running speed arrives at $380 \mathrm{~km} / \mathrm{h}$.

Since the turbulence flows around train are more disturbances with the increased speed, the flow energies are converted to aerodynamic drag, noise and vibrations. Many design problems which have been neglected at low train speeds are being raised, such as aerodynamic noise, structural vibration due to fluid/structure interaction, impulse pressure waves as two trains passing-by each other and singletrain or two trains running in tunnels, and ear discomfort of passengers inside train, etc. [1-2]. Aerodynamic designs on the new generation of high-speed trains must consider these major limitation factors, therefore aerodynamic design on China new high-speed trains becomes one of key techniques.

In the paper, aerodynamic design on China new high speed trains of CRH380A and 380B are briefly introduced. In fact, for the aerodynamic design of CRH380A, we mainly evaluated the aerodynamic performances for numerous design models and gave the optimum selection. For the aerodynamic design of CRH380B, we mainly explored the drag deduction for the optimizations of different local structures. 


\section{Aerodynamic Design on CRH380A}

\subsection{Design Models}

Twenty train-head models with the length of $12 \mathrm{~m}$ were designed, by considering the numerous limitation conditions, such as the range of pilot view, the equipment installation, the inside space and manufacturing cost etc., and by selected the geometrical design variables, such as the fore-body length, the horizontal- and longitudinal- sectional shapes and areas, the longitudinal changeable ratio of cross-sectional areas, nose shape and lateral cover shapes of bogie region etc.. Figure 1(a-e) only shows their five train-head design models and Figure 1(f) gives the comparison of the longitudinal-sectional shapes of the five models. Model 5 likes a sword and the differences of other models behave mainly at nose shapes and pilot visual regions.

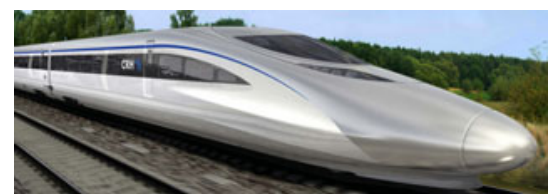

(a)Model 1

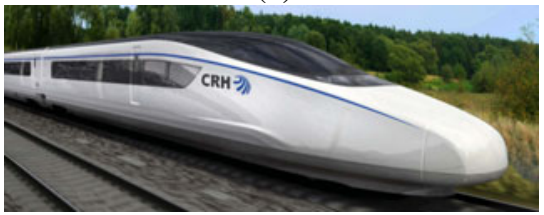

(c)Model 3

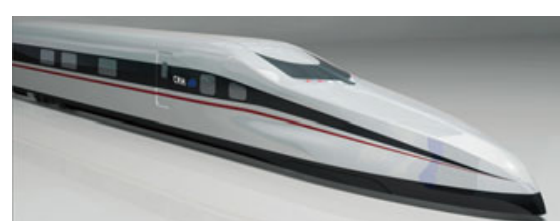

(e)Model 5

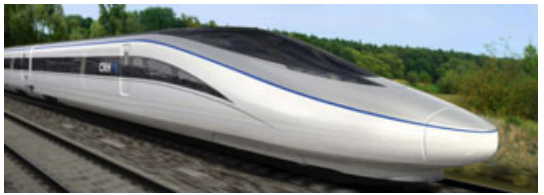

(b)Model 2

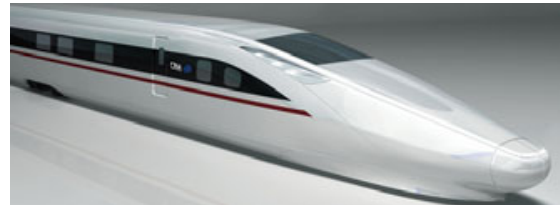

(d)Model 4

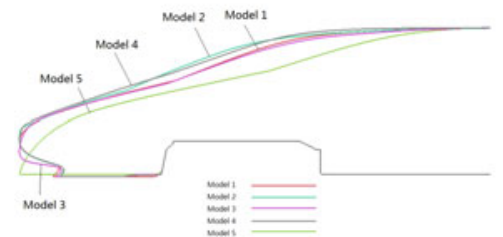

(f) Longitudinal sectional shapes

Fig. 1 Five train-head models and sectional-shape comparison

\subsection{Aerodynamic Performance}

In general, the desirable China new high-speed trains should be aerodynamically stable and have low aerodynamic forces. It is well know that the aerodynamic drag is proportional to the square of speed, while the mechanical drag is proportional to the speed. Compared with the mechanical drag, the aerodynamic drag occupies $90 \%$ larger of the total drag as the train speed exceeding of $300 \mathrm{~km} / \mathrm{h}$, thus, low aerodynamic drag design is the most important issue for the new generation 
high-speed train design. Comparing with the aeronautical vehicles, the train length is very long and runs close to the ground, considering the wheel/rail relation and running stability, the aerodynamic lifts of train fore- and after-bodies should also be lower.

The cross-wind can make the train to produce the lateral forces and the overturn moments to influence the safe travelling, thus, the lateral forces and the overturn moments should also be lower.

First, in the open air without any cross-wind effects, the aerodynamic drag and lift coefficients were analyzed with numerical simulation for all of the twenty design models. The calculations were carried out for these models with the foreand after-bodies of train and one middle-body at a train speed of $350 \mathrm{~km} / \mathrm{h}$. In here, only the drag and lift coefficients for the above five models are presented in the Figure 2 and Figure 3, respectively. The models of 1, 3 and 5 have the lower total drag coefficient. The lift coefficients of the fore- and middle-bodies are negative and the after-body is positive lift, but model 1 has the lowest after-body lift coefficient and model 5 has the highest after-body lift coefficient. We can preliminary presume that the model 1 is the best selection for the new generation of China high-speed train.

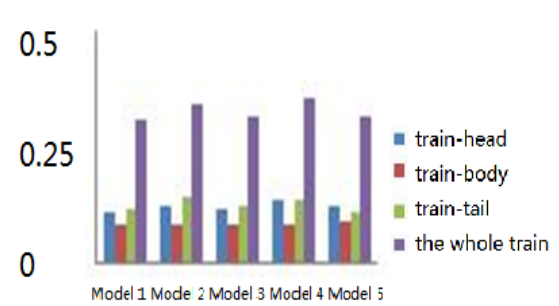

Fig. 2 Drag coefficients of different models

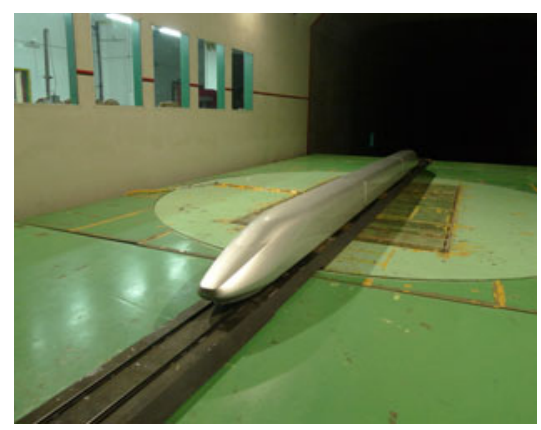

Fig. 4 Wind tunnel model

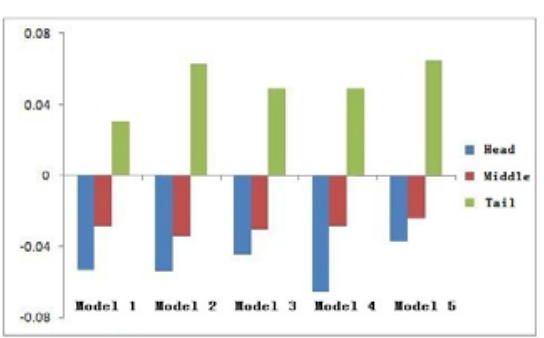

Fig. 3 Lift coefficients of different models

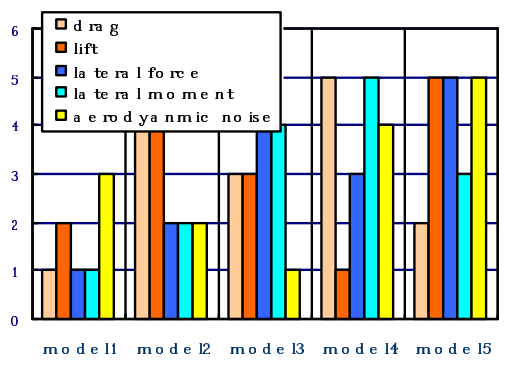

Fig. 5 Comparison of aerodynamic coefficients 
Then, the reduced scale wind tunnel models were designed and tests were done at the speed of $60 \mathrm{~m} / \mathrm{s}$ and yaw angles from -30 degree to 30 degree. The drag, lift and moment coefficients, pressure distribution and aerodynamic noise etc. were measured. Figure 4 shows the wind tunnel test model. The comparison of nondimensional integrated aerodynamic performance for the five models is shown in Figure 5, which contains total drag, after-body lift, fore-body lateral force, forebody pitching moment, fore-body lateral moment and aerodynamic noise. It indicates that model 1 is the best in the five models. The high-speed train of CRH380A running in the railway of Beijing-Shanghai line was produced based on model 1.

Then, since the two-trains passing-by in the open air or in tunnel and singletrain running in tunnel cannot be simulated with wind tunnel tests, the complex running conditions can produce the impulse pressure waves and influence the structural strength of travelling train and passenger's comfort. We use numerical techniques to evaluate the amplitudes of positive and negative impulse pressure waves. The impulse waves are dependent on the speed of running trains, the shape of train fore- and after-bodies, train length and width, the distance between track lines, tunnel length and tunnel cross-sectional area. In here, the tunnel length and cross-sectional area are taken as $2000 \mathrm{~m}$ and $100 \mathrm{~m}^{2}$, the train length and the running speed are $400 \mathrm{~m}$ and $300 \mathrm{~km} / \mathrm{h}$. Figure 6 and Figure 7 present the comparison of the maximum of the impulse positive pressure and the minimum of the absolute impulse negative pressure for the above three complex cases, respectively. Two-trains passing-by each other in tunnel produce the larger positive and negative impulse pressure values, which are about 2100pa to 2600pa and -4900pa to -5200pa, respectively. Single-train passing by in tunnel produces the positive impulse pressure about $800 \mathrm{pa}$ to $1000 \mathrm{pa}$, and negative impulse pressure about -2800 pa to -3200 pa. Two-trains passing by each other in open air produce the positive impulse pressure about 700pa to 900pa, and negative impulse pressure about -900pa to -1400pa.

The structural strength of the original high-speed train of CRH2 was designed based on the amplitude of impulse pressure waves of $4000 \mathrm{~Pa}$ and the new highspeed train of CRH380A is improved to 6000pa to satisfy the requirement of twotrains passing by in tunnel.

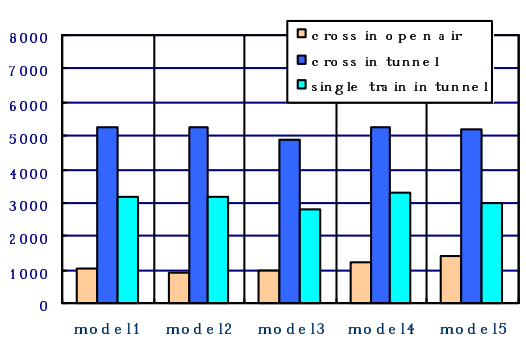

Fig. 6 Positive pressure maximum

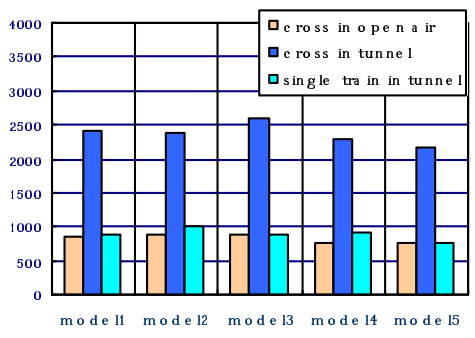

Fig. 7 Absolute negative pressure minimum 
Finally, the real high-speed train of CRH380A with eight cars (total length $200 \mathrm{~m})$ at the speed $350 \mathrm{~km} / \mathrm{h}$ was analyzed in the open air without cross-wind shown in Figure 8. The first pantograph locates at the fourth-car, and the second pantograph at sixth-car. The drag and lift distributions for different cars are shown in Figure 9. The fourth- and sixth-cars with pantographs have the largest drag than other cars, and the fore- and after-bodies also have the larger drag. Lift coefficients from the first- to seventh-cars are negative, however, the after-body positive. Relatively, the fore- and after-bodies have the absolute maximum drags.

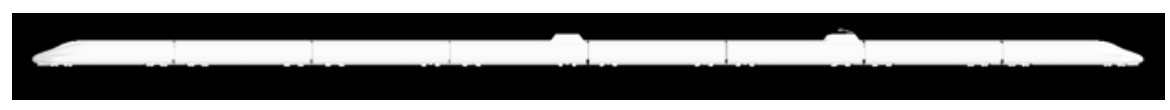

Fig. 8 Eight-car model

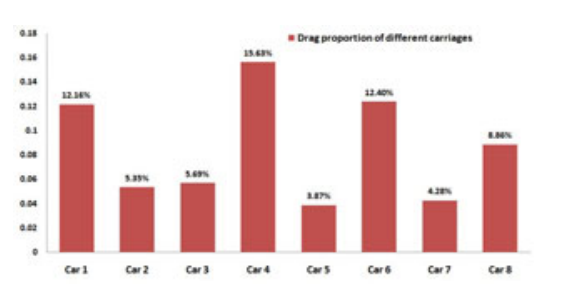

(a)

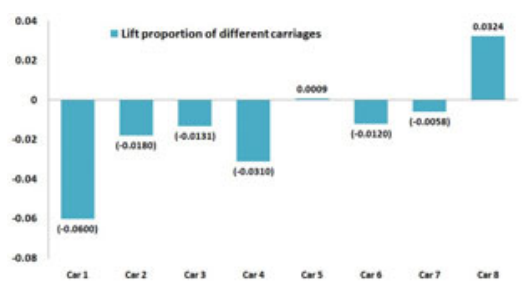

(b)

Fig. 9 Drag and lift distribution for different car

\section{Aerodynamic Design on CRH380B}

CRH3 was introduced into China from Siemens, which can run with the speed of $300 \mathrm{~km} / \mathrm{h}$. In order to speed up to $350 \mathrm{~km} / \mathrm{h}$, aerodynamic design on drag deduction is mainly concerned.

\subsection{Aerodynamic Drag Distribution of CRH3}

First, the aerodynamic drag distributions of the original $\mathrm{CRH} 3$ train with eight cars (total length $200 \mathrm{~m}$ ) were calculated at the $350 \mathrm{~km} / \mathrm{h}$ in the open air without any cross-wind effects. The train model for calculation is shown in Figure 10, which contains the complex bogies, pantographs and their covers, joint parts between two cars, ventilation covers etc. Aerodynamic drag distribution from the train nose to tail along the longitudinal direction is given in Figure 11. It indicates the drag contributions are produced mainly from fore-and after-bodies, joint parts between two cars, two pantographs, ventilation covers. For drag deduction optimization, these structures should be modified. 
Fig. 10 CRH3 model with eight cars

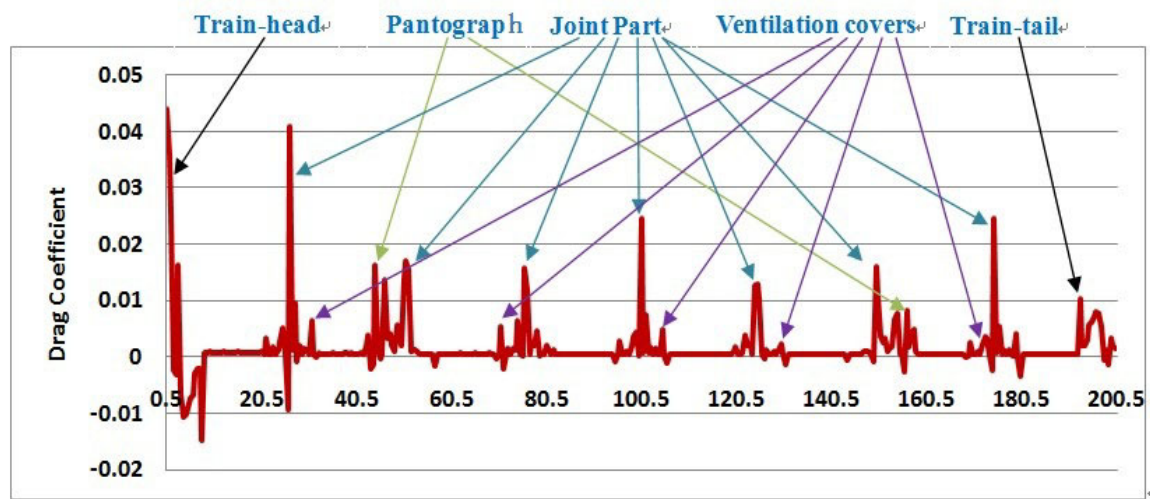

Fig. 11 Aerodynamic drag distribution along longitudinal direction $(\Delta \mathrm{l}=0.5 \mathrm{~m})$

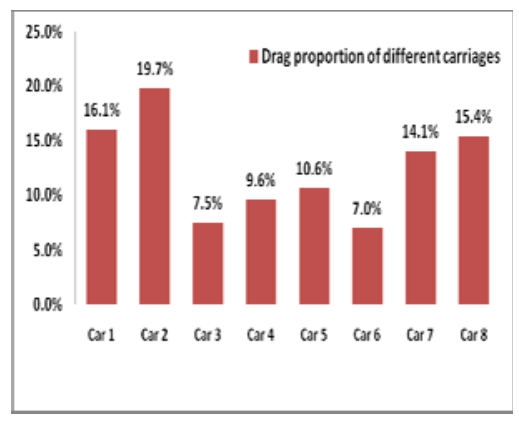

Fig. 12 Drag distribution of cars

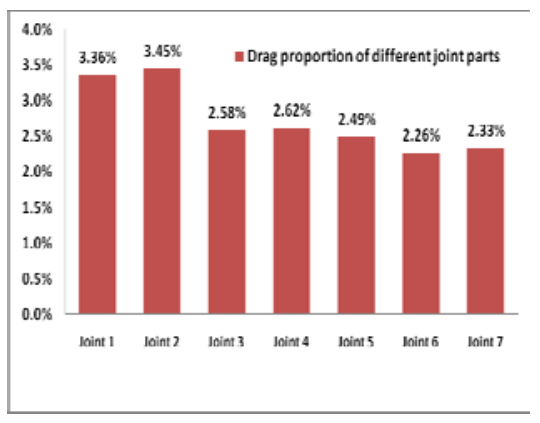

Fig. 13 Drag distribution of joint parts

The percentage ratio of each car to total drag of the train is shown in Figure 12. The aerodynamic drag of fore- and after-bodies occupies $31.5 \%$ of the total drag, $33.8 \%$ of the total drag for the second- and seventh-cars with two pantographs, and $34.7 \%$ for the other four cars. Every joint part between two cars contributes to the total drag is presented in Figure 13. The drag sum of all joint parts is about $19 \%$ of the total drag. The first- and second- joint parts are produced much larger aerodynamic drag than other parts. The drag distributions of bogies and their installation regions are shown in Figure 14, whose sum contributes $27.4 \%$ to the total drag. Bogies drags are smaller than that of their installation regions. The first installation region of bogie has the maximum drag contribution to the total drag. We also evaluate the drag distributions of other parts, in which the drag of two 


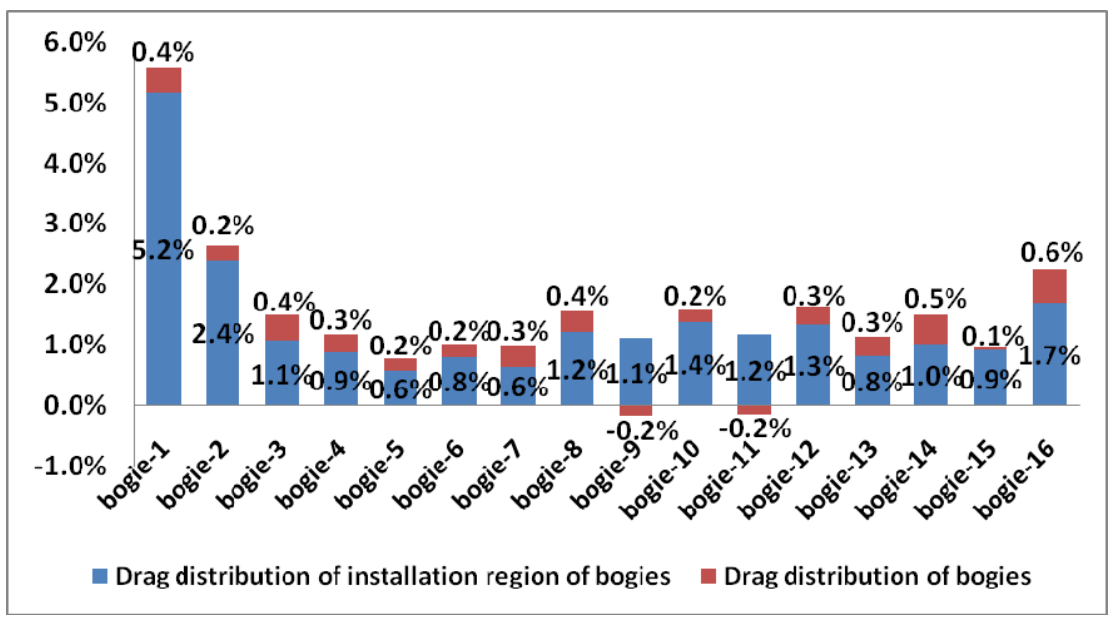

Fig. 14 Drag distribution of bogies and their installation region

pantographs and their covers is about $12 \%$ of the total drag, and all ventilation covers is about $7.6 \%$ of the total drag of the train. From these analyses, the aerodynamic drag distributions of every part of the train are known, thus the drag deduction should be optimized for these parts with larger drag.

\subsection{Local Drag Deduction Design of CRH3}

Through the above analyses, keeping the fore- and after-bodies, pantographs and bogies unchangeable, the following structures are suggested to be optimized: (1) adding outer joint parts between cars shown in Fig, 15(a); (2) modified the

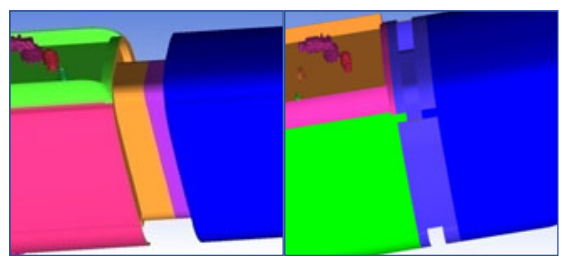

(a) Joint part between two cars

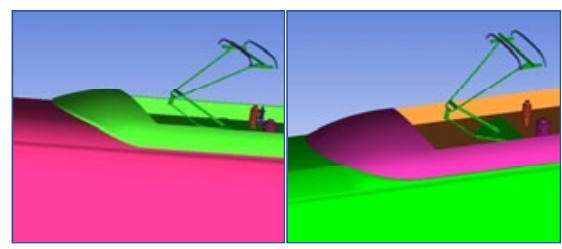

(c) Pantograph cover

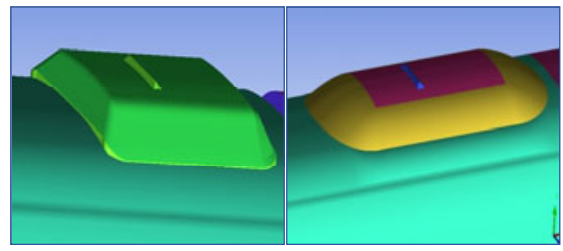

(b) Ventilation cover

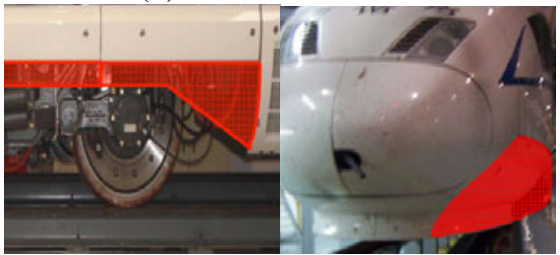

(d) Region of bogies

Fig. 15 Local modification parts of CRH3 
streamline ventilation covers shown in Figure 15(b); (3) modified the streamline pantograph covers shown in Figure 15(c); (4) enwrapping the first bogie and adding the skirts for other bogies shown in Figure 15(d).

\subsection{Wind Tunnel Tests for Local Modified Model}

Under the wind speed of $60 \mathrm{~m} / \mathrm{h}$ and 1:8 reduced scale models with three cars, the wind tunnel tests were also done to check the drag deduction effects for different optimization structures. The effects of drag deduction are shown in Table 1. All local structural modifications are benefit for the aerodynamic drag deduction and the integrated optimization model 8 can reduce the total aerodynamic drag of $8.9 \%$.

Table 1 The effect of drag deduction

\begin{tabular}{|l|l|l|}
\hline Model & Modified description & Drag \\
\hline 1 & Adding half outer joint parts between two cars & $-4.1 \%$ \\
\hline 2 & Adding full outer joint parts between two cars & $-4.2 \%$ \\
\hline 3 & Streamline ventilation covers & $-1.4 \%$ \\
\hline 4 & Streamline pantograph covers and without ventilation covers & $-4.6 \%$ \\
\hline 5 & Adding 80 mm skirts for all bogie region & $-0.9 \%$ \\
\hline 6 & $5+$ only half skirt for first bogie region & $-1.7 \%$ \\
\hline 7 & 5+only full wrapping skirt for first bogie & $-5.7 \%$ \\
\hline 8 & $1+3+5+$ streamline pantograph covers & $-8.9 \%$ \\
\hline
\end{tabular}

\subsection{Aerodynamic Performance of CRH380B}

After the above wind tunnel tests, the optimized local structures are obtained for the model of three cars. We need further to know the effect of drag reduction for the real running train with eight cars. Numerical simulations are again used for the evaluation of aerodynamic performance for the optimized train. The comparison of aerodynamic drag distribution along the longitudinal direction is given in Figure 16. Local drag deductions are obvious in the optimization regions. Figure 17 shows the comparison of drag distribution for eight cars. The drag deduction for different cars behaves the anti-symmetric change, namely, the drag deductions of CRH380B are the second-car, the third-car, the fourth-car and the eighth-car, however the drag augmentations are the corresponding cars of the seventh-car, the sixth-car, the fifth-car and the first-car. The second-car with pantograph and its adjacent third-car, along with tail-train have obvious the effect for drag deduction. The decreased values of these cars are larger than the increased values of the corresponding anti-symmetric cars. The total aerodynamic drag of CRH380B decreases $8.67 \%$ than the original train of CRH3. The target of drag deduction is achieved. 


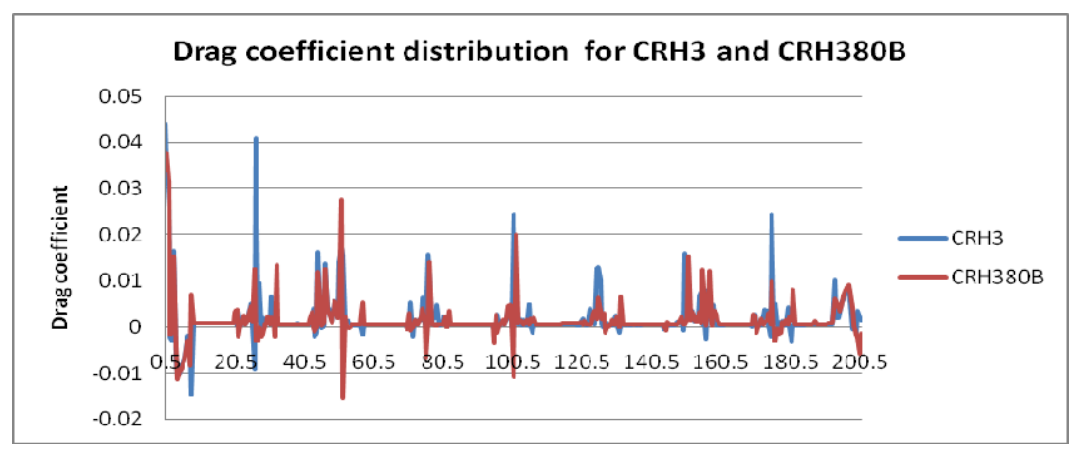

Fig. 16 Aerodynamic drag distribution for CRH3 and CRH380B

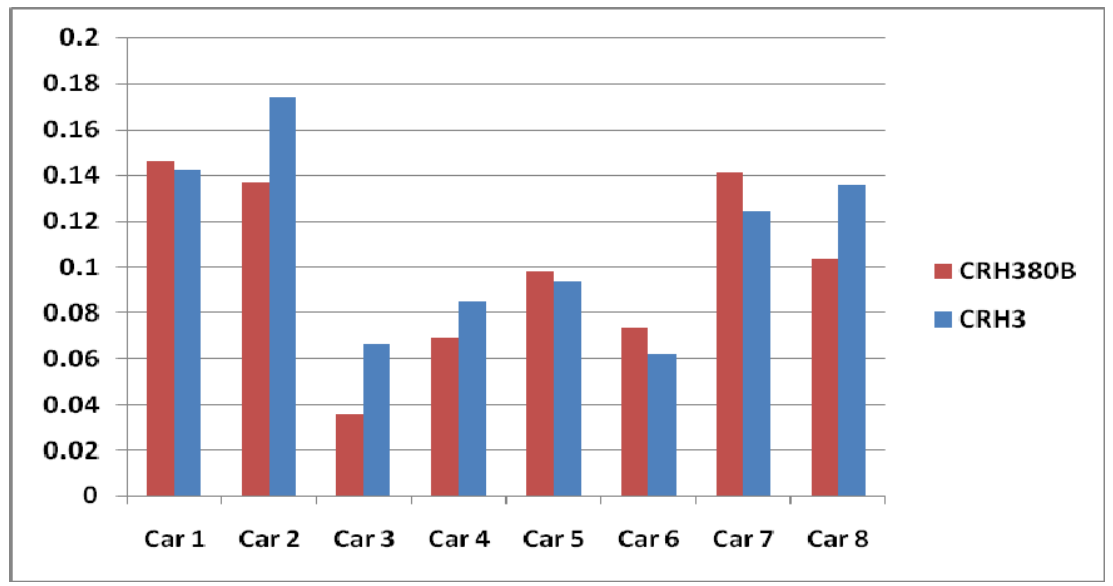

Fig. 17 Aerodynamic drag distribution of cars

\section{Conclusions}

By numerical simulations and wind-tunnel tests, the new generation of high-speed train of CRH380A are determined based on aerodynamic performances from 20 train-head models designed under the consideration of geometrical variables and engineering limitation conditions. The running CRH380A train in BeijingShanghai line testifies it has excellent aerodynamic performance.

By the analyses of aerodynamic drag distribution of $\mathrm{CRH} 3$, the train parts of drag deduction are found. By the wind-tunnel tests, local optimized structures for drag reduction are validated. Then the effect of drag deduction for the real train of CRH380B with eight-cars is calculated and compared with the original train of CRH3. The running CRH380B train in Beijing-Shanghai line also shows it has excellent aerodynamic performance. 


\section{References}

Raghunathan, R.S., Kim, H.-D., Setoguchi, T.: Aerodynamics of high-speed railway train. Progress in Aerospace Sciences 38, 469-514 (2002)

Schetz, J.A.: Aerodynamics of high-speed trains. Annual Review of Fluid Mechanics (33), 371-414 (2001) 